\title{
High-contrast imaging testbed
}

K. Baker, D. Silva, L. Poyneer, B. Macintosh, B.

Bauman, D. Palmer, T. Remington, M. Delgadillo-Lariz

February 4, 2008

SPIE Photonics West

San Jose, CA, United States

January 19, 2008 through January 24, 2008 
This document was prepared as an account of work sponsored by an agency of the United States government. Neither the United States government nor Lawrence Livermore National Security, LLC, nor any of their employees makes any warranty, expressed or implied, or assumes any legal liability or responsibility for the accuracy, completeness, or usefulness of any information, apparatus, product, or process disclosed, or represents that its use would not infringe privately owned rights. Reference herein to any specific commercial product, process, or service by trade name, trademark, manufacturer, or otherwise does not necessarily constitute or imply its endorsement, recommendation, or favoring by the United States government or Lawrence Livermore National Security, LLC. The views and opinions of authors expressed herein do not necessarily state or reflect those of the United States government or Lawrence Livermore National Security, LLC, and shall not be used for advertising or product endorsement purposes. 


\title{
High-contrast imaging testbed
}

\author{
K.L. Baker, D.A. Silva, L.A. Poyneer, B.S. Macintosh, B.J. Bauman, D. Palmer, T.P. Remington \\ and M.A. Delgadillo-Lariz \\ Lawrence Livermore National Laboratory, 7000 East Ave., Livermore, CA, USA 94550
}

\begin{abstract}
Several high-contrast imaging systems are currently under construction to enable the detection of extra-solar planets. In order for these systems to achieve their objectives, however, there is considerable developmental work and testing which must take place. Given the need to perform these tests, a spatially-filtered Shack-Hartmann adaptive optics system has been assembled to evaluate new algorithms and hardware configurations which will be implemented in these future high-contrast imaging systems. In this article, construction and phase measurements of a membrane "woofer" mirror are presented. In addition, results from closed-loop operation of the assembled testbed with static phase plates are presented. The testbed is currently being upgraded to enable operation at speeds approaching $500 \mathrm{hz}$ and to enable studies of the interactions between the woofer and tweeter deformable mirrors.
\end{abstract}

Keywords: Adaptive optics, high contrast, Shack-Hartmann wave-front sensor, spatial filter

\section{INTRODUCTION}

Future adaptive optics systems on ground-based telescopes will enable direct observation of extra-solar Jovian planets. The observation of the reflected or emitted spectra from these planets at high spectral resolution will provide valuable scientific information regarding their atmospheric constituents which is unattainable with current techniques used to study these planets such as the Doppler-shift of or intensity variation in the parent stars emission. Likewise, these instruments will study such planet populations at distances which are farther away from the parent star than current techniques have thus-far achieved and as such are complimentary to the techniques mentioned above. There are several attractive options for spatially-filtered wave-front sensors on these adaptive optics systems including pyramid sensors in direct phase mode ${ }^{1}$, interferometers ${ }^{2}$ and Shack-Hartmann wave-front sensors ${ }^{3}$. The first generation of these instruments, such as the Gemini Planet Imager(GPI), will employ a spatially-filtered Shack-Hartmann wave-front sensor. ${ }^{4}$ In order for this instrument, as well as future instruments, to be successful, a number of algorithms and hardware configurations must be tested in a laboratory setting before the implementation of these instruments on telescopes can take place.

In this article we report on the development of a spatially-filtered Shack-Hartmann wave-front sensor which will be used to test the algorithms and hardware configurations that will be implemented on the Gemini Planet Imager. The testbed, along with its components, is described in detail in section 2. The results from running the testbed in closed-loop mode with static phase plates is presented in section 3 and the results are summarized in section 4 of this article.

\section{EXPERIMENTAL LAYOUT}

The optical layout of the high-contrast laboratory breadboard system is shown below in Figure 1. The testbed consists primarily of a spatially-filtered Shack-Hartmann wavefront sensor, a MEMS-based spatial-light-modulator functioning as a "tweeter" built by the Boston Micromachines Corporation (BMC), a membrane mirror functioning as a "woofer", a 1 mrad tip-tilt stage manufactured by Piezosystems Jena, a light source, a wave-front aberrator, a far-field camera and computer hardware/software to analyze the wave-front and implement the phase correction. The system is at present controlled using the IDL programming language which is currently running the system at about $1 \mathrm{hz}$. Because of this relatively slow speed, the results presented below represent a correction of the 1024 actuator MEMS device with stationary phase plates. The membrane mirror and tip-tilt stage have been tested separately in an interferometer but have not yet been integrated into the adaptive optics testbed. A description of the membrane mirror is given in greater detail in section 2.1 below. As currently configured, the testbed can be run with either a single mode fiber-coupled HeNe laser at $632 \mathrm{~nm}$ or a superluminescent diode at $680 \mathrm{~nm}$ with a bandwidth of $8 \mathrm{~nm}$ for its light source. For the results presented below, a HeNe laser was utilized as the light source. 
The system aperture is set by the MEMS mirror. The "tweeter" deformable mirror utilized for this testbed was one of the early devices with a continuous face sheet coated with gold. As such there are several actuators scattered across the surface of the MEMS device that do not respond to voltages sent to the actuators. As a consequence, a small diameter was used on the MEMS mirror to preclude dead actuators within the aperture of the system, thereby enabling a high Strehl ratio to be achieved. In particular, the image of the input aperture on the MEMS device represents a sixteen actuator diameter circle. The actuator pitch on the MEMS mirror is $340 \mu \mathrm{m}$ such that the system aperture is $5.4 \mathrm{~mm}$ imaged onto the MEMS device and $10.9 \mathrm{~mm}$ at the actual aperture of the system.

The system is relay-imaged between the various deformable mirrors. In particular, the system aperture, location of the ground level phase plate, is relay-imaged onto the "woofer" mirror/tip-tilt stage(currently a flat mirror) which is in turn relay-imaged onto the MEMS "tweeter" mirror. The MEMS mirror is then relay-imaged onto the lenslet array after passing through the spatial filter to remove frequencies beyond the control of the MEMS device. The Hartmann spots are then relay-imaged onto the Photometrics wave-front camera.

The wave-front sensor itself is a spatially-filtered Shack-Hartmann wave-front sensor. The lenslet array used for the Hartmann wave-front sensor has a pitch of $100 \mu \mathrm{m}$ and was manufactured by Vitrum Technology, LLC. The wave-front camera has 128 by 128 pixels each of which is $24 \mu \mathrm{m}$ square. A $4 \times 4$ pixel area on the wave-front camera is used for each of the Hartmann spots such that the relay imaging system between the lenslet array and the wave-front camera is operating at a magnification ratio of 0.96 . The spatial filter is placed on a kinematic mount between the lenses imaging the MEMS mirror onto the lenslet array. The reference spots for the wave-front sensor are formed by placing a pinhole in the location of the spatial filter.

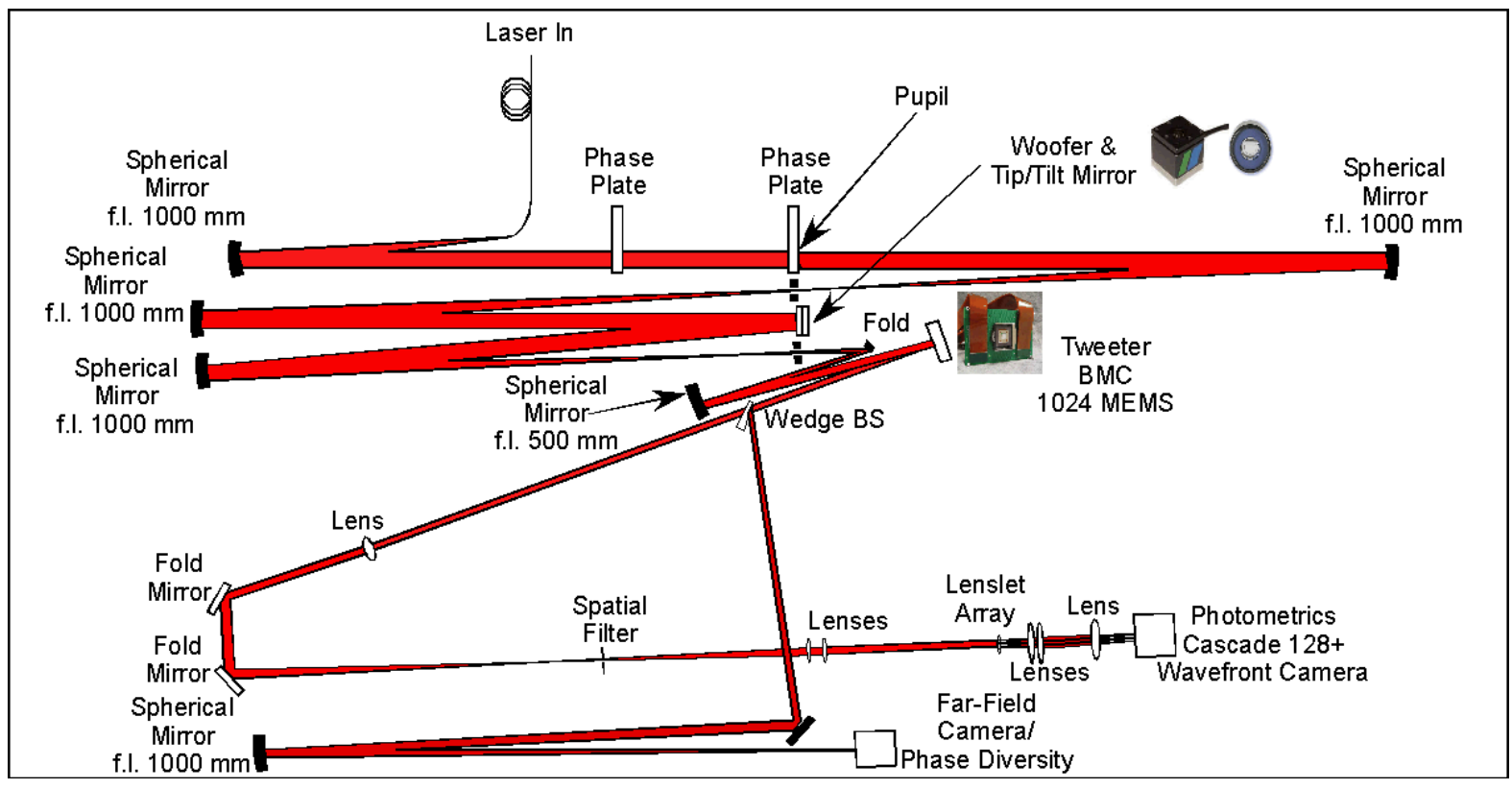

Fig. 1. Laboratory breadboard setup used to test the performance of the adaptive optics algorithms in a controlled laboratory environment. This configuration represents a spatially-filtered Shack-Hartmann wave-front sensor with a woofer and tweeter deformable mirror. 


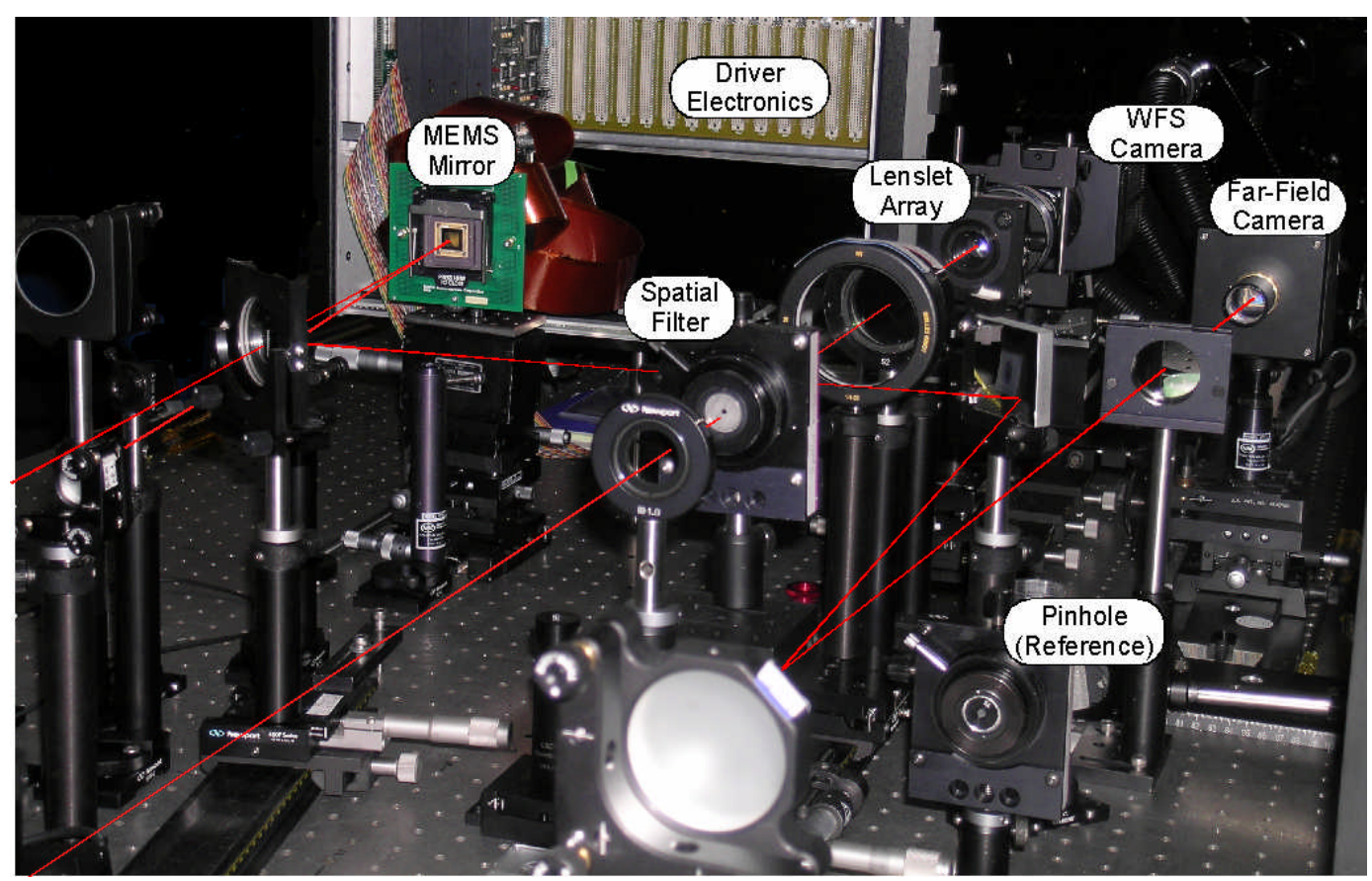

Fig. 2. This figure represents an actual picture of the testbed with various components labeled. The laser path is drawn on top of the picture.

\subsection{Woofer mirror}

For the "woofer", a membrane deformable mirror was designed and assembled using a commercial aluminized pellicle beamsplitter for the membrane and an etched PCB board for the electrode structure. ${ }^{5}$ A drawing of the etched actuator structure on the PCB board is shown in Fig. 3a below. An actual picture of the completed membrane mirror is then given in Fig. 3b. Given our ability to customize the actuator geometry for the woofer mirror, we will be better able to test the effects of alignment between the woofer and tweeter actuators in future experiments.

(a)
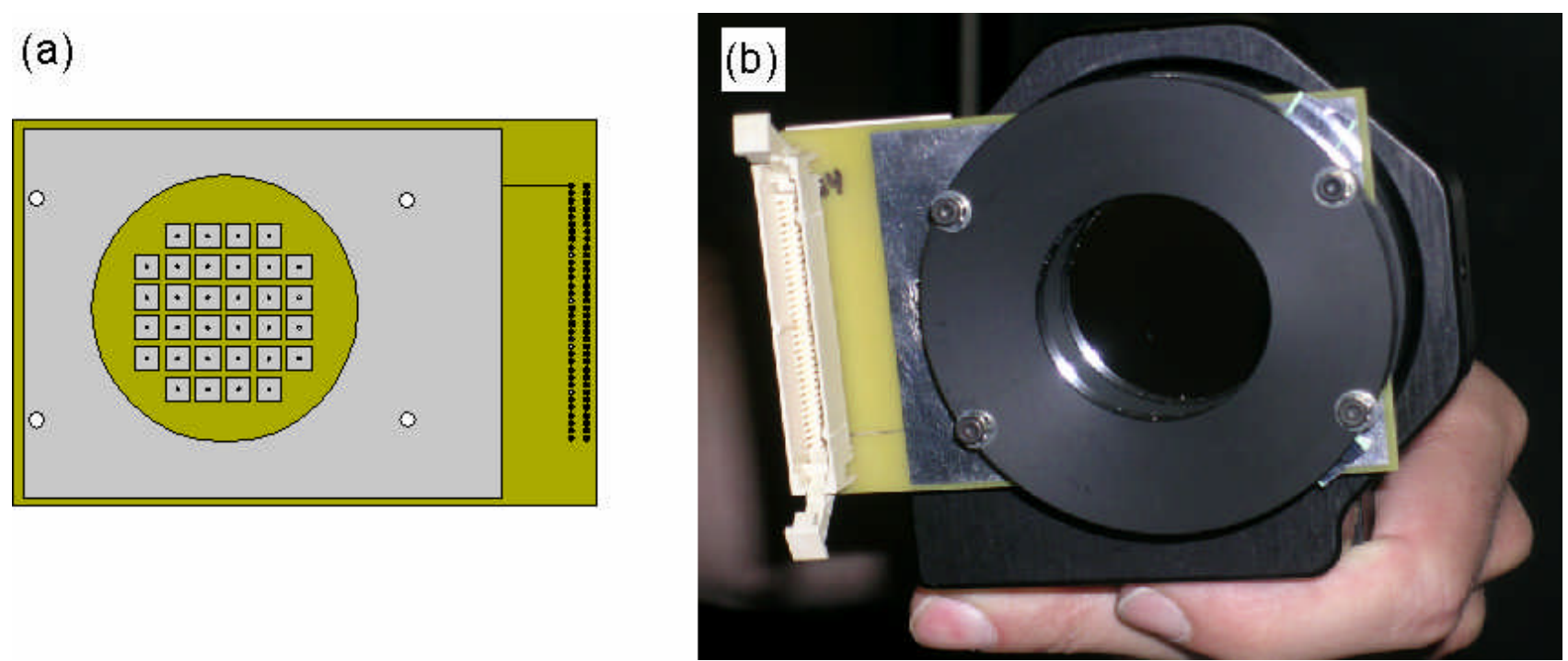

Fig. 3. This figure represents both a drawing of the electrode array, Fig. 3a, as well as an actual picture of the woofer mirror assembled for the testbed, Fig. $3 b$. 
The approach taken to perform the initial characterization of the membrane mirror was to set up a Twyman-Green interferometer as the characterization instrument. A tilt is placed on the reference leg of the Twyman-Green interferometer such that a large number of fringes, $>50$, are apparent in the interference pattern on the CCD camera. Fourier transforming the interference pattern produces a Fourier spectrum, which has a DC component and an up and down-shifted satellite structure due to the large carrier frequency introduced by the applied tilt on the reference leg. By filtering all but the up-shifted satellite structure and shifting this structure back to the origin, the phase can be determined. ${ }^{6}$ The wrapped phase calculated in this manner is equal to the inverse tangent of the imaginary part divided by the real part of the inverse Fourier transform. The wrapped phase was then unwrapped using a Fourier reconstructor. This unwrapped phase is then used to build up a phase response vs. amplitude curve for each of the actuators on the membrane mirror.

The reconstructed phase for four of the center actuators, when $150 \mathrm{~V}$ is applied in turn to each of the four actuators in the woofer mirror, is shown in Fig. 4. The maximum optical phase excursion for these four actuators is approximately five waves.

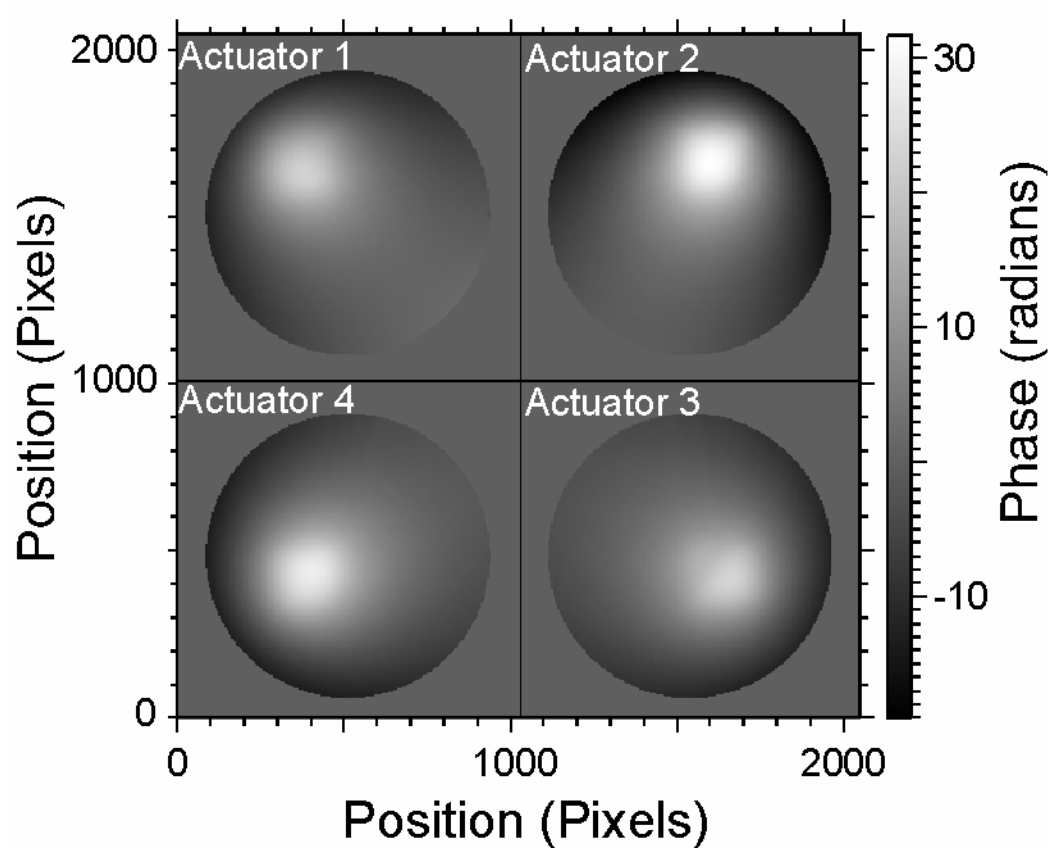

Fig. 4. This figure represents the reconstructed phase for four of the center actuators, when $150 \mathrm{~V}$ is applied in turn to each of the four actuators in the woofer mirror.

\subsection{Phase Plates}

The phase plates were designed to provide a reasonable scaling with the atmosphere expected on the Gemini telescope. The phase plates for the testbed were based on a two layer atmospheric model for Cerro Pachon calculated using the Arroyo code. ${ }^{7}$ The two layer model assumed that the atmosphere was composed of a layer at zero height which was traveling at $5 \mathrm{~m} / \mathrm{sec}$ and contained $81 \%$ of the turbulence level. A second layer at $8.9 \mathrm{~km}$ travels at $26 \mathrm{~m} / \mathrm{sec}$ and contains $19 \%$. The overall turbulence level assumed was a Fried parameter of $r_{o}=0.24 \mathrm{~m}$ at $750 \mathrm{~nm}$. The instrument on the Gemini telescope will have 44 actuators across an $8 \mathrm{~m}$ aperture or a subaperture spacing of $\mathrm{d}=0.18 \mathrm{~m}$. The wave-front fitting error will be proportional to $\left(\mathrm{d} / \mathrm{r}_{\mathrm{o}}\right)^{5 / 3}$. For the instrument on the Gemini telescope, $\mathrm{d} / \mathrm{r}_{\mathrm{o}} \sim 0.76$ based on the subaperture spacing and the overall Fried parameter. For the testbed, 16 actuators will be used over an aperture of 10.88 $\mathrm{mm}$ for a subaperture spacing of $0.68 \mathrm{~mm}$. The Fried parameter for the testbed should then be $\mathrm{r}_{\mathrm{o}}=0.9 \mathrm{~mm}$ at $632 \mathrm{~nm}$. The two phase plates were designed to give an overall Fried parameter of $1.17 \mathrm{~mm}$ at $680 \mathrm{~nm}$. The upper phase plate was designed to be $3.3 \mathrm{~mm} \sim(1.17 \mathrm{~mm})(0.19)^{5 / 3}$ and the lower phase plate designed to be $1.3 \mathrm{~mm} \sim(1.17 \mathrm{~mm})(0.81)^{5 / 3}$. The time delay error is proportional to $v \tau / \mathrm{r}_{\mathrm{o}}$ where $\mathrm{v}$ is the velocity of the atmospheric layer and $\tau$ is the time delay between measurement and correction. Assuming a wind speed of $5 \mathrm{~m} / \mathrm{sec}$, a Fried parameter of $0.24 \mathrm{~m}$ and a time delay of $1 /(2000 \mathrm{hz})$ at the telescope, the testbed velocity should be $\sim 3.65 \mathrm{~mm} / \mathrm{sec}$ assuming a Fried parameter of $1.17 \mathrm{~mm}$ and a 
time delay of $1 /(500 \mathrm{hz})$. The frequency that the plates would spin would then be $\sim 0.7$ rotations/minute. The scintillation index is proportional to $\lambda \mathrm{L} / \mathrm{r}_{\mathrm{o}}{ }^{2}$ where $\mathrm{L}$ is the height of the upper level and $\lambda$ is the wavelength. To match the scintillation index of the Gemini telescope, the upper level phase plate should be placed $0.3 \mathrm{~m}$ upstream of the aperture. That will enable the testbed to operate under similar conditions of wave-front fitting error, time delay and scintillation index as expected on the Gemini telescope.

Two Kolmogorov phase plates were designed at Lawrence Livermore National Laboratory. The phase plates were designed to have Fried parameters of $1.31 \mathrm{~mm}$ and $3.33 \mathrm{~mm}$ as detailed above. The simulated structure function, along with an analytic fit, are shown below in Fig. 5. Fig. 5a represents the ground layer atmospheric phase plate with a Fried parameter of $1.31 \mathrm{~mm}$ and Fig. $5 \mathrm{~b}$ represents the upper layer atmospheric phase plate with a Fried parameter of 3.33 $\mathrm{mm}$. These plates were designed with $20 \mu \mathrm{m}$ square pixels with an overall phase plate size of 4096 by 4096 pixels. The two phase plates were then manufactured by Lexitech, Inc. using computer numerical control machining of optical plastic in combination with an index-matching optical epoxy placed between the plastic and two glass plates placed on either side. ${ }^{8}$
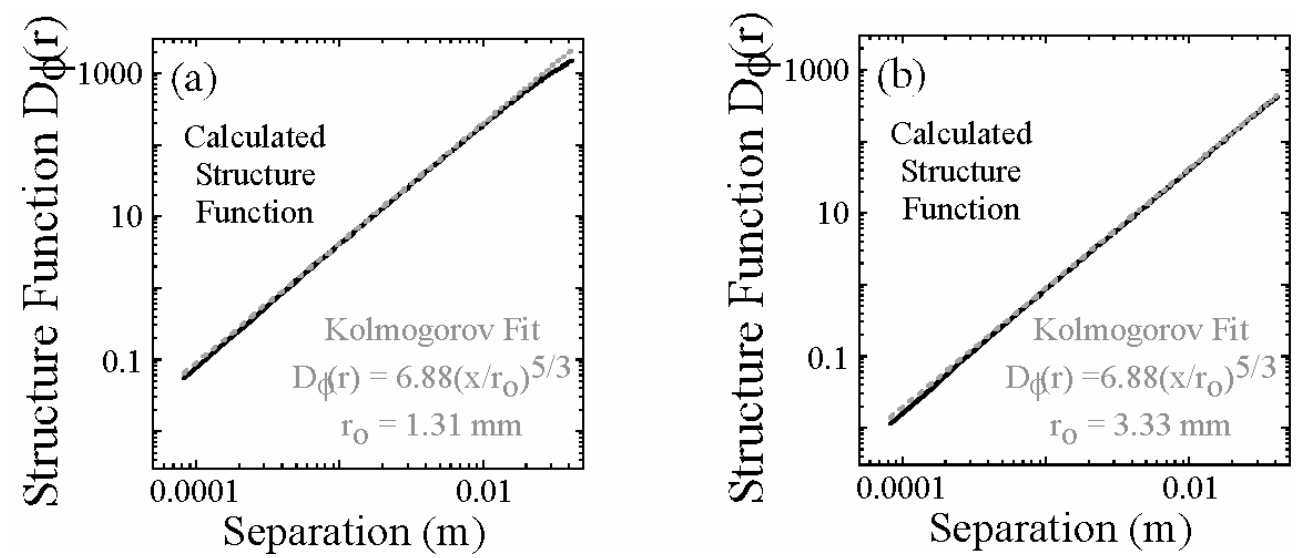

Fig. 5. This figure represents the structure function of the two phase plates. Analytic fits to the calculated structure function are shown as dashed gray lines. The phase plate with the largest level of turbulence is shown in Fig. 4a and corresponds to a Fried parameter of $r_{0}=1.31 \mathrm{~mm}$. The second phase plate, represented in Fig. 4b, has a lower level of turbulence and corresponds to a Fried parameter of $r_{0}=3.33 \mathrm{~mm}$.

\section{PERFORMANCE WITH STATIC PHASE PLATES}

The performance of the tested with the tweeter mirror was tested with both of the phase plates described above. In both cases the testbed was run in closed-loop for fifty iterations and the far-field was taken at the end of the fifty iterations. The Hartmann spot locations were determined using a centroider with a Gaussian weighting function ${ }^{910}$. A pinhole placed in the location of the spatial filter was used to generate the reference wave-front. An iteratively-weighted centroider was used to determine the locations of each of the reference centroids. ${ }^{10}$ A centroider with a Gaussian weighting function, $\mathrm{w}(\mathrm{x}, \mathrm{y})$, was then used in closed-loop which contained both a "global" $\sigma$ (root-mean-square deviation of position from the mean), as well as the "local" centroid locations $\left(\mathrm{x}_{\mathrm{c}}\right.$ and $\left.\mathrm{y}_{\mathrm{c}}\right)$,

$$
w(x, y)=\frac{1}{2 \pi \sigma^{2}} e^{-\frac{1}{2 \sigma^{2}}\left(x-x_{c}\right)^{2}} e^{-\frac{1}{2 \sigma^{2}}\left(y-y_{c}\right)^{2}} .
$$

With the slope measurements determined by the centroider, a Fourier reconstructor ${ }^{11}$ was used to reconstruct the phase. For both phase plates, a gain of 0.25 was used when applying the calculated correction to the deformable mirror.

The closed-loop results for the upper atmosphere phase plate, $r_{o}=3.33 \mathrm{~mm}$, placed at the ground layer position is shown in Fig. 6. The initial far-field, lower left image in Fig. 6, is dominated primarily by the cylindrical phase error of the uncorrected MEMS device itself. The in-band residual error, as measured by the filtered Shack-Hartmann wave-front sensor, is shown in the top left of Fig. 6. This residual error approaches the $1 \mathrm{~nm}$ root-mean-square(RMS) error after approximately 25 iterations with a gain of 0.25 . The final far-field is shown in the lower left of Fig. 6 . This represents a saturated image of the far-field which shows the structure. The MEMS device itself is causing a periodic structure in the 
far-field which can be seen near the edge of the far-field image in a cross pattern. The radial average of an unsaturated far-field is shown in the upper right and is used to estimate the Strehl ratio achieved. The Strehl ratio calculated in this manner is $\mathrm{S}_{\mathrm{r}}=0.92$.
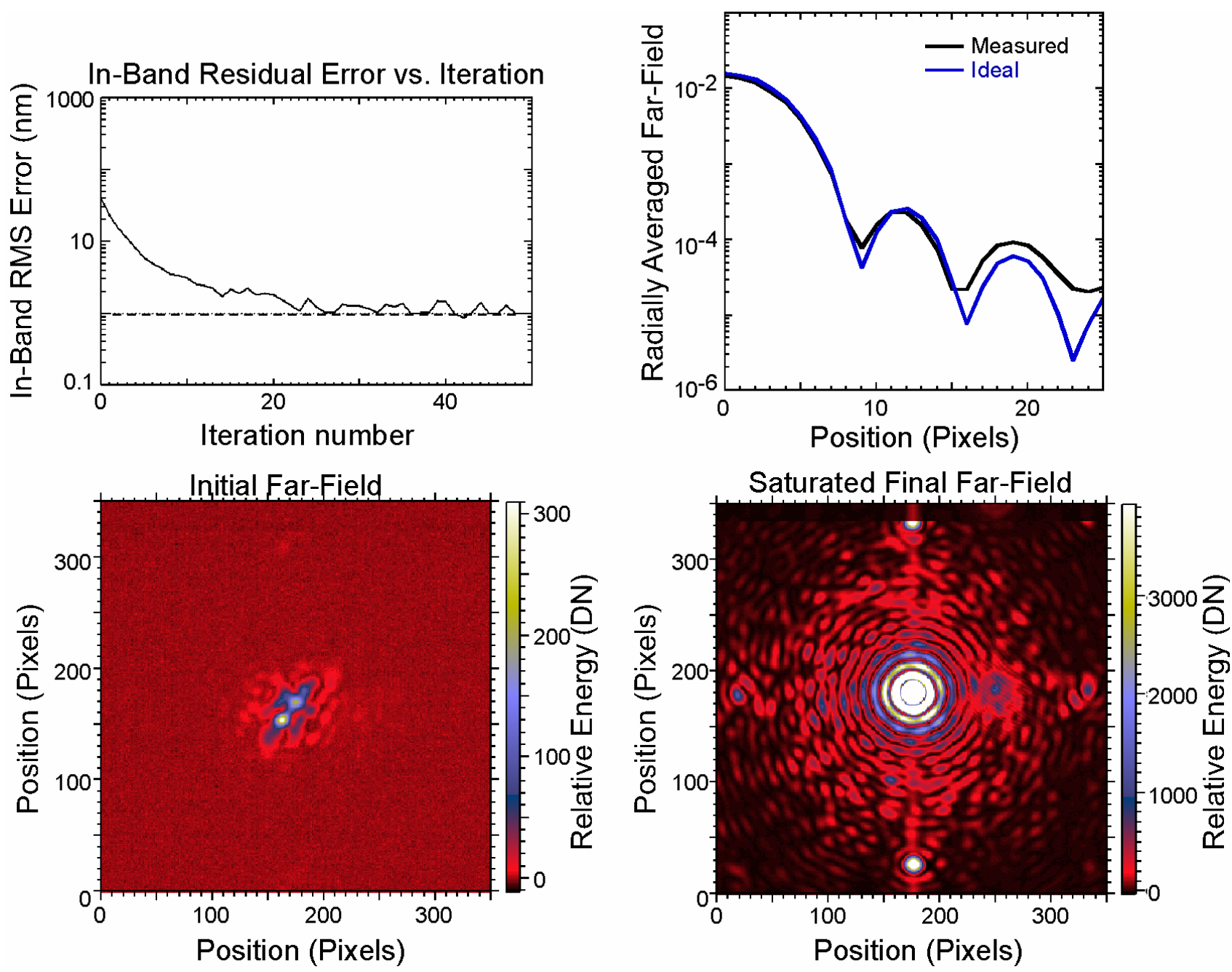

Fig.6. This figure represents the closed-loop results for the tweeter mirror correction of the $r_{0}=3.33 \mathrm{~mm}$ phase plate.

The closed-loop results for the ground layer atmosphere phase plate, $r_{o}=1.31 \mathrm{~mm}$, is shown in Fig. 7. The initial farfield, lower left image in Fig. 6, is dominated in this case primarily by the Kolmogorov turbulence spectrum from the phase plate. The in-band residual error, as measured by the filtered Shack-Hartmann wave-front sensor, is shown in the top left of Fig. 6. This residual error approaches the $1 \mathrm{~nm}$ RMS error after approximately 40 iterations with a gain of 0.25 . The final far-field is shown in the lower left of Fig. 6. This represents a saturated image of the far-field which shows the structure. Again the MEMS device itself is causing a periodic structure in the far-field which can be seen near the edge of the far-field image in a cross pattern. The radial average of an unsaturated far-field is shown in the upper right and is used to estimate the Strehl ratio achieved. The Strehl ratio calculated in this manner is $\mathrm{S}_{\mathrm{r}}=0.78$. 

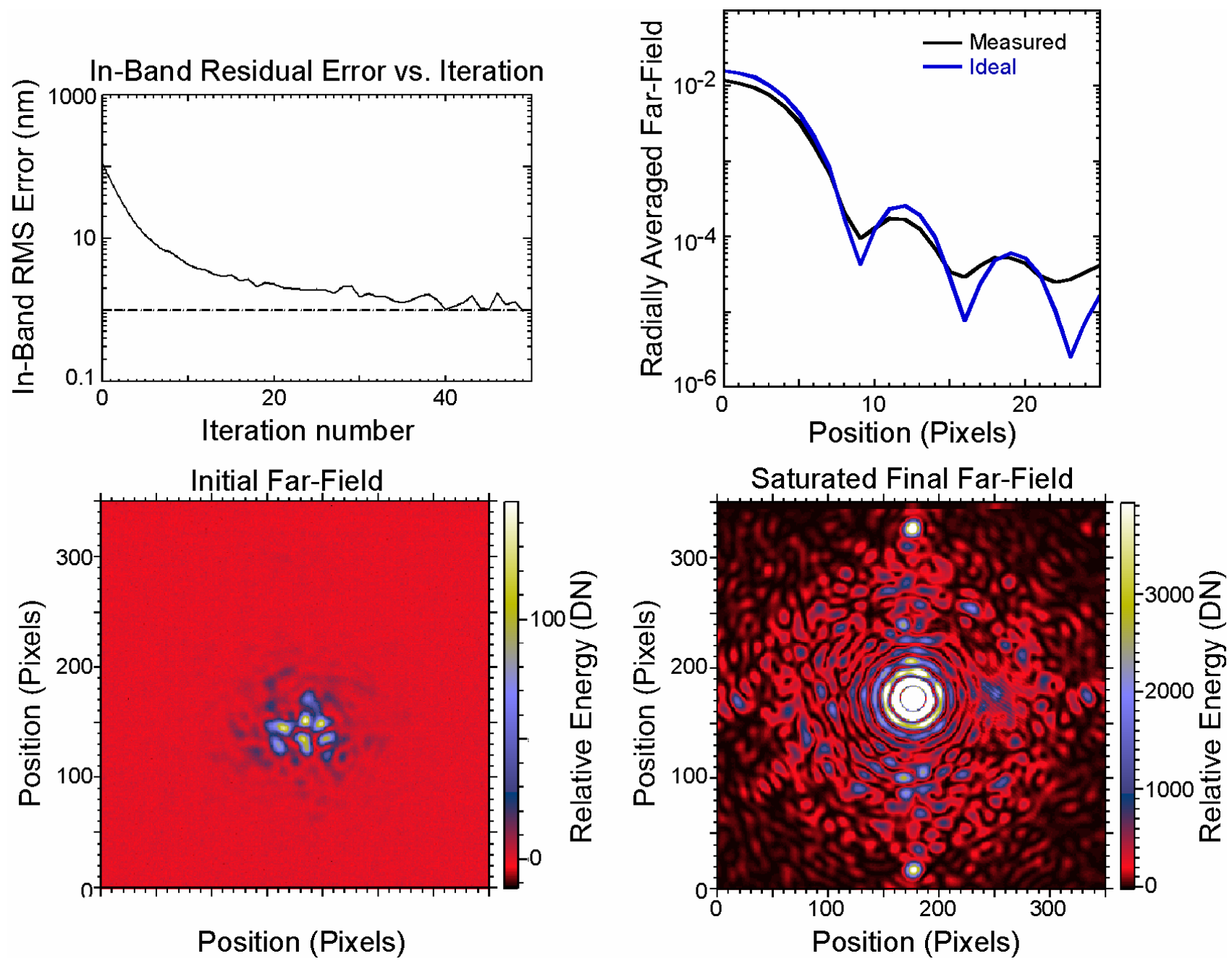

Fig. 7. This figure represents the closed-loop results for the tweeter mirror correction of the $r_{0}=1.31 \mathrm{~mm}$ phase plate.

\section{SUMMARY}

A breadboard for testing algorithms and hardware configurations for high-contrast imaging has been developed. This process has included the development of inexpensive and customizable woofer membrane mirrors whose actuator pitch can be configured to be a multiple of the actuator pitch for the MEMS. This process also involved the development of Kolmogorov phase plates which will enable the breadboard to be run under similar conditions of fitting error, time delay and scintillation as experienced on the Gemini telescope. Given the testbeds current speed, initial closed-loop tests were performed with stationary phase plates. The results from running the testbed on static phase plates have demonstrated that the testbed can achieve high Strehl ratios, $\mathrm{Sr}>0.9$, and low in-band residual RMS errors, $\sim 1 \mathrm{~nm}$.

A number of changes are being implemented to the testbed, both software and hardware, to make the system better able to test algorithms and to verify experimental results. The algorithms are currently being converted from IDL into C code such that the system will soon be able to run at approximately $500 \mathrm{hz}$, the read-out rate of the unbinned Photometrics wave-front sensor camera. Once the conversion has taken place, the phase plates will be rotated to simulate wind speed as the system is run in closed-loop. This will allow for testing algorithms for predictive correction of the turbulence layers. Likewise, the membrane mirror and tip-tilt stage are currently being integrated into the adaptive optics testbed. This will allow testing of algorithms controlling the mode sharing between the woofer and tweeter. An apodizing mask will also be added to the testbed to suppress the diffraction of the far-field within the controllable band of MEMS mirror to enable the contrast ratio to be accurately measured and to provide an additional measurement of the in-band residual 
error. In addition a phase-retrieval diagnostic will be added to correct for non-common path errors between the Hartmann wave-front sensor and the far-field diagnostic

\section{ACKNOWLEDGEMENTS}

The authors would like to thank the Center for Adaptive Optics and the Boston Micromachines Corporation for lending us the BMC 1024 actuator MEMS device. This work was performed under the auspices of the U.S. Department of Energy by Lawrence Livermore National Laboratory in part under Contract W-7405-Eng-48 and in part under Contract DE-AC52-07NA27344.

\section{REFERENCES}

[1] Donald W. Phillion and Kevin Baker, "Two-sided pyramid wavefront sensor in the direct phase mode," Proc. SPIE 6272, 627228 (2006).

[2] K.L. Baker, "Interferometric Wavefront Sensors for High Contrast Imaging Optics Express," 14, 10970 (2006).

[3] Lisa A. Poyneer and Bruce Macintosh, "Spatially filtered wave-front sensor for high-order adaptive optics," J. Opt. Soc. Am. A 21 (5), 810 (2004).

[4] Bruce Macintosh, James Graham, David Palmer, Rene Doyon, Don Gavel, James Larkin, Ben Oppenheimer, Leslie Saddlemyer, J. Kent Wallace, Brian Bauman, Julia Evans, Darren Erikson, Katie Morzinski, Donald Phillion, Lisa Poyneer, Anand Sivaramakrishman, Remi Soummer, Simon Thibault, and Jean-Pierre Veran, "The Gemini Planet Imager," Proc. SPIE 6272, 62720L-1 (2006).

[5] M.M. Moallem and K.L. Baker, "Low-Cost Compact Adaptive Optics Systems," Report No. F071-006-0373, 2006.

[6] Malgorzata Kujawinska, in [Interferogram Analysis], edited by David W Robinson and Graeme T Reid (Institute of Physics Publishing, Bristol and Philadelphia, 1993).

[7] Mitch Troy (private communication).

[8] Steven M. Ebstein, "Psuedo-random phase plates," Proc. SPIE 4493, 150 (2002).

[9] M. Nicolle, T. Fusco, G. Rousset, and V. Michau, "Improvement of Shack-Hartmann wave-front sensor measurement for extreme adaptive optics," Optics Letters 29, 2743 (2004).

[10] K.L. Baker and M.M Moallem, "Iteratively Weighted Centroiding for Shack-Hartmann Wave-front Sensors Optics Express," 15, 5147 (2007).

[11] Lisa A. Poyneer, Donald T. Gavel, and James M. Brase, "Fast wave-front reconstruction in large adaptive optics systems with use of the Fourier transform," J. Opt. Soc. Am. A 19 (10), 2100 (2002). 